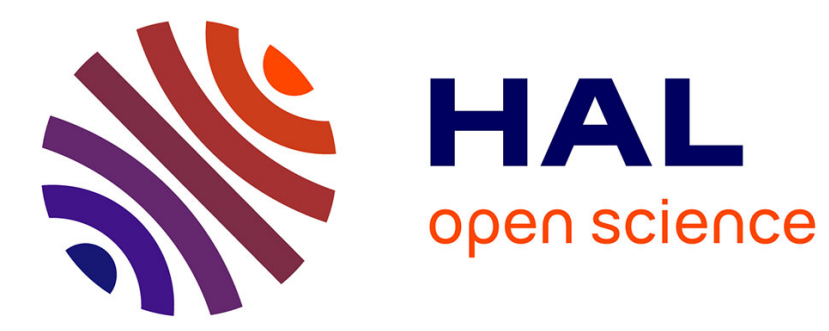

\title{
Asymptotics in Knuth's parking problem for caravans
} Jean Bertoin, Grégory Miermont

\section{To cite this version:}

Jean Bertoin, Grégory Miermont. Asymptotics in Knuth's parking problem for caravans. Random Structures and Algorithms, 2006, 29 (1), pp.38-55. 10.1002/rsa.20092 . hal-00004229

\section{HAL Id: hal-00004229 \\ https://hal.science/hal-00004229}

Submitted on 10 Feb 2005

HAL is a multi-disciplinary open access archive for the deposit and dissemination of scientific research documents, whether they are published or not. The documents may come from teaching and research institutions in France or abroad, or from public or private research centers.
L'archive ouverte pluridisciplinaire HAL, est destinée au dépôt et à la diffusion de documents scientifiques de niveau recherche, publiés ou non, émanant des établissements d'enseignement et de recherche français ou étrangers, des laboratoires publics ou privés. 


\title{
Asymptotics in Knuth's parking problem for caravans
}

\author{
Jean Bertoin $*$ Grégory Miermont
}

10th February 2005

\begin{abstract}
We consider a generalized version of Knuth's parking problem, in which caravans consisting of a random number of cars arrive at random on the unit circle. Then each car turns clockwise until it finds a free space to park. Extending a recent work by Chassaing and Louchard [8], we relate the asymptotics for the sizes of blocks formed by occupied spots with the dynamics of the additive coalescent. According to the behavior of the caravan's size tail distribution, several qualitatively different versions of eternal additive coalescent are involved.
\end{abstract}

Keywords: Parking problem, additive coalescent, bridges with exchangeable increments.

M.S.C. code: $60 \mathrm{~F} 17,60 \mathrm{~J} 25$.

*LPMA, Université Paris 6, 175 rue du Chevaleret, F-75013 Paris

'DMA École Normale Supérieure. 45, rue d'Ulm, 75230 Paris Cedex 05, France 


\section{Introduction}

The original parking problem of Knuth can be stated as follows. Consider a parking lot with $n$ spaces, identified with the cyclic group $\mathbb{Z} / n \mathbb{Z}$. Initially the parking lot is empty, and $m \leq n$ cars in a queue arrive one by one. Car $i$ tries to park on a uniformly distributed space $L_{i}$ among the $n$ possible, independently of other cars, but if the space is already occupied, then it tries places labeled $L_{i}+1, L_{i}+2, \ldots$ until it finally finds a free spot to park. As cars arrive, blocks of consecutive occupied spots are forming. It appears that a phase transition occurs at the stage where the parking lot is almost full, more precisely when the number of free spots is of order $\sqrt{n}$. Indeed, while the largest block of occupied spots is of order $\log m$ with high probability as long as $\sqrt{m}=o(n-m)$, a block of size approximately $n$ is present (while the others are of order at most $\log n$ ) with high probability when $n-m=o(\sqrt{m})$. In the meanwhile, precisely when $n-m$ is of order $\lambda \sqrt{m}$ with $\lambda>0$, a clustering phenomenon occurs as $\lambda$ decays. The behavior of this clustering process has been studied precisely by Chassaing and Louchard [8]. It turns out that the process of the relative sizes of occupied blocks is related to the so-called standard additive coalescent [10, 1].

The model originates from a problem in Computer Science: spaces in the parking lot should be thought of as elementary memory spaces, each of which can be used to store elementary data (cars). Roughly, our aim in this work is to investigate the more general situation where one wants to store larger files, each requiring several elementary memory spaces. In other words, single cars are replaced by caravans of cars, i.e. several cars may arrive simultaneously at the same spot. In this direction, it will be convenient to consider a continuous version of the problem, that goes as follows. Let $p_{1}, \ldots p_{m}$ be a sequence of positive real numbers with sum 1 , and $s_{1}, \ldots, s_{m}, m$ distinct locations on the unit circle $\mathbb{T}:=\mathbb{R} / \mathbb{Z}$. Imagine that $m$ drops of paint with masses $p_{1}, \ldots, p_{m}$, fall successively at locations $s_{1}, \ldots, s_{m}$. Each time a drop of paint falls, we brush it clockwise in such a way that the resulting painted portion of $\mathbb{T}$ is covered by a unit density of paint. So at each step the drop of paint is used to cover a new portion of the circle and the total length of the painted part of the circle when $i \leq m$ drops have fallen is $p_{1}+\cdots+p_{i}$. In this setting, drops of paint play the role of caravans, and the painted portion of the circle corresponds to occupied spots in the parking lot.

More precisely, we consider an increasing sequence $\left(A_{0}, \ldots, A_{m}\right)$ of open subsets of $\mathbb{T}$, starting from $A_{0}=\varnothing$ and ending at $A_{m}=\mathbb{T}$, which can be thought of as the successive painted portions of the circle. Given $A_{i}$ and the location $s_{i+1}$ from where the $i+1$-th drop of paint will be brushed, we paint as many space as possible to the right of $s_{i+1}$ with the quantity $p_{i+1}$ of paint, without covering the already painted parts, i.e. the blocks of $A_{i}$. Alternatively, we break the $i+1$-th caravan into several pieces, so that to fill as much as possible the holes left by $\mathbb{T} \backslash A_{i}$ after $s_{i+1}$, when reading in clockwise order. The last car to park arrives at some location $t_{i+1}$, and we let $A_{i+1}$ be the union of $A_{i}$ and the arc between $s_{i+1}$ and $t_{i+1}$, see Figure 1. More formal definitions will come in Sect. 2.

In particular, $A_{i}$ is a disjoint union of open intervals and $\operatorname{Leb}\left(A_{i}\right)=p_{1}+\ldots+p_{i}$. Let $\Lambda^{\mathbf{p}}(i)\left(=\Lambda\left(p_{1}, \ldots, p_{m}, s_{1}, \ldots, s_{m}, i\right)\right)$ be the sequence of the Lebesgue measures of the connected components of $A_{i}$, ranked by decreasing order. It will be convenient to view $\Lambda^{\mathbf{p}}(i)$ as an infinite sequence, by completing with an infinite number of zero terms.

Now consider the following random problem. Let $\ell>0$ be a random variable with 


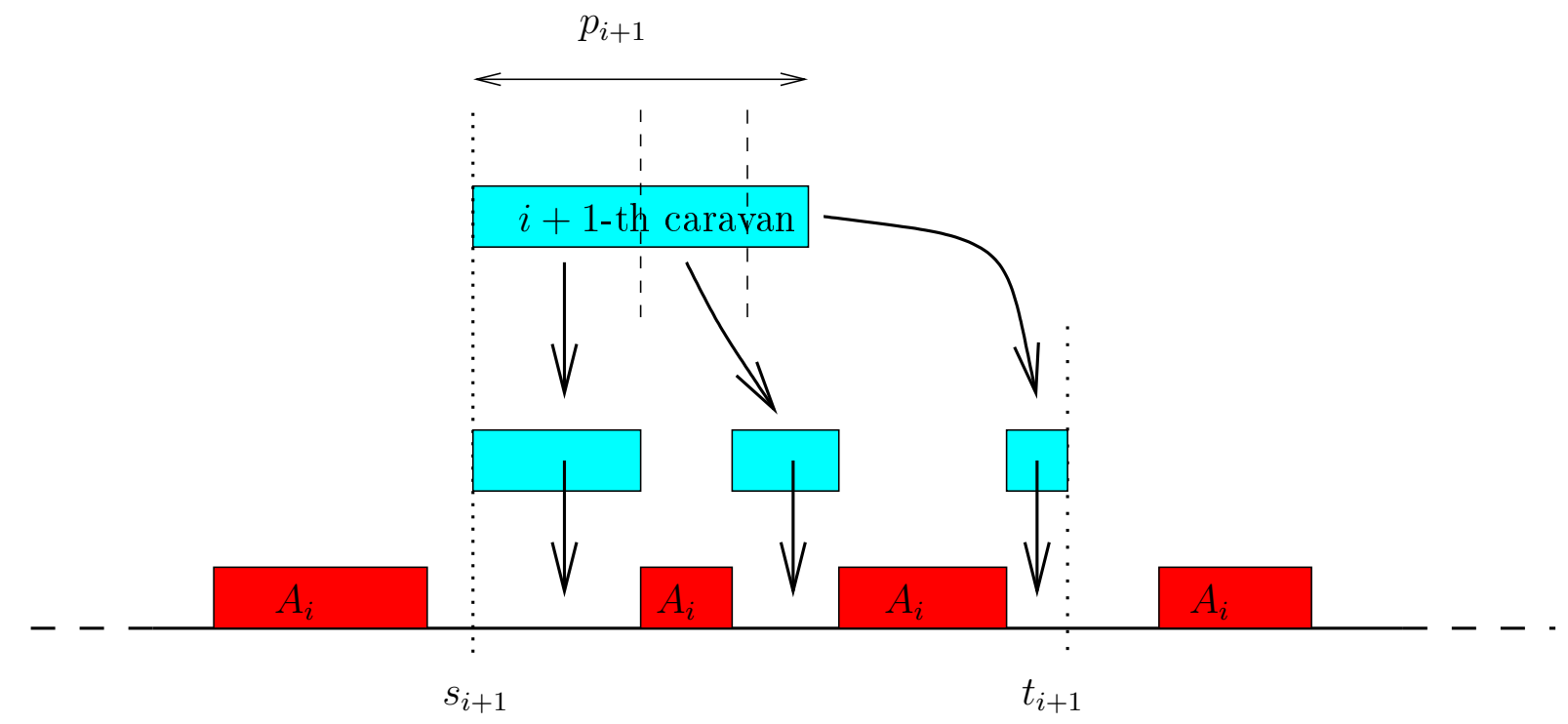

Figure 1: Arrival, splitting and parking of the $i+1$-th caravan in the process

finite expectation $\mu_{1}=\mathbb{E}[\ell]$. We say that $\ell \in \mathcal{D}_{2}$ whenever $\ell$ has a finite second moment $\mu_{2}=\mathbb{E}\left[\ell^{2}\right]$. For $\alpha \in(1,2)$, we say that $\ell \in \mathcal{D}_{\alpha}$ whenever

$$
\mathbb{P}(\ell>x) \underset{x \rightarrow \infty}{\sim} c x^{-\alpha}
$$

for some $0<c<\infty$. This implies that $\ell$ is in the domain of attraction of a spectrally positive stable random variable with index $\alpha$, and we stress that our results can be extended under this more general hypothesis; (11) is only intended to make things easier. We suppose from now on that $\ell \in \mathcal{D}_{\alpha}$ for some $\alpha \in(1,2]$, and take a random iid sample $\ell_{1}, \ell_{2}, \ldots$ of variables distributed as $\ell$, and independently of this sequence, iid uniform random variables on $[0,1), U_{1}, U_{2}, \ldots$ For $\varepsilon>0$, set

$$
T_{\varepsilon}=\inf \left\{i: \ell_{1}+\ldots+\ell_{i} \geq 1 / \varepsilon\right\},
$$

so by the elementary renewal theorem, $T_{\varepsilon} \sim 1 /\left(\varepsilon \mu_{1}\right)$. Then introduce the sequence $\left(\ell_{i}^{*}, 1 \leq i \leq T_{\varepsilon}\right)$ defined by

$$
\ell_{i}^{*}=\ell_{i} \text { for } 1 \leq i \leq T_{\varepsilon}-1 \text { and } \ell_{T_{\varepsilon}}^{*}=\varepsilon^{-1}-\left(\ell_{1}+\ldots+\ell_{T_{\varepsilon}-1}\right),
$$

so the terms of $\ell^{*}$ sum to $1 / \varepsilon$.

Following Chassaing and Louchard [8], we are interested in the formation of macroscopic painted components in the limit when $\varepsilon$ tends to 0 , at times close to $T_{\varepsilon}$, i.e. when the circle is almost entirely painted. Specifically, we let

$$
\mathbf{X}^{(\varepsilon)}(t)=\Lambda^{\mathbf{p}}\left(T_{\varepsilon}-\left\lfloor t \varepsilon^{-1 / \alpha}\right\rfloor\right), \quad t \geq 0,
$$

for $\Lambda^{\mathbf{p}}$ defined as above with the data $m=T_{\varepsilon}, p_{i}=\varepsilon \ell_{i}^{*}, s_{i}=U_{i}$. Observe that $T_{\varepsilon}-\left[t \varepsilon^{-1 / \alpha}\right]$ decreases when $t$ increases, and therefore, in order to investigate the formation of painted components, we should consider the process $\left(\mathbf{X}^{(\varepsilon)}(t), t \geq 0\right)$ backwards in time. This is what we shall do in Theorem 1, using the exponential time change $t \rightarrow e^{-t}$. 
Before describing our main result, let us first recall some features of the additive coalescent. The additive coalescent $\mathbf{C}$ is a Markov process with values in the infinite ordered simplex

$$
S=\left\{\mathbf{s}=\left(s_{1}, s_{2}, \ldots\right): s_{1} \geq s_{2} \geq \ldots \geq 0, \sum_{i=1}^{\infty} s_{i} \leq 1\right\}
$$

endowed with the uniform distance, whose evolution is described formally by: given that the current state is $\mathbf{s}$, two terms $s_{i}$ and $s_{j}, i<j$, of $\mathbf{s}$ are chosen and merge into a single term $s_{i}+s_{j}$ (which implies some reordering of the resulting sequence) at a rate equal to $s_{i}+s_{j}$. A version $(\mathbf{C}(t), t \in \mathbb{R})$ of this process defined for times describing the whole real axis is called eternal. We refer to [1, 3] for background.

As shown in [4], eternal additive coalescents can be encoded by certain bridges. Specifically, let $B=(B(x), 0 \leq x \leq 1))$ be a càdlàg real-valued process with exchangeable increments, such that $B(0)=B(1)=0$. Suppose further that $B$ has infinite variation and no negative jumps a.s. Then $B$ attains its overall infimum at a unique location $V$ (which is uniformly distributed on $[0,1]$ ), and $B$ is continuous at $V$. Consider the so-called Vervaat transform which maps the bridge $B$ into an excursion $\mathcal{E}$ defined by

$$
\mathcal{E}(x)=B(V+x)-B(V) \text { for } 0 \leq x \leq 1,
$$

where the addition $V+x$ is modulo 1 . Finally, we let for $t \geq 0$

$$
\mathcal{E}_{x}^{(t)}=\mathcal{E}(x)-t x \quad \text { for } 0 \leq x \leq 1,
$$

and introduce $\mathbf{F}(t)$ as the random element of $S$ defined by the ranked sequence of the lengths of the constancy intervals of the process $\underline{\mathcal{E}}^{(t)}=\left(\inf _{0 \leq y \leq x} \mathcal{E}^{(t)}(y), 0 \leq x \leq 1\right)$. Here, a constancy interval means a connected component of the complement of the support of the Stieltjes measure $\mathrm{d}(-\underline{\mathcal{E}})$. Finally, if we define $\mathbf{C}(t)=\mathbf{F}\left(e^{-t}\right)$, then $\mathbf{C}=(\mathbf{C}(t),-\infty<$ $t<\infty)$ is an eternal additive coalescent (see Section 6.1 for comments and details).

In this work, eternal additive coalescent associated to certain remarkable bridges will play a key role. More precisely, we write $\mathbf{C}^{(2)}=\left(\mathbf{C}^{(2)}(t),-\infty<t<\infty\right)$ for the eternal additive coalescent $\mathbf{C}$ constructed above when $B=\mathcal{B}^{(2)}$ is a standard Brownian bridge; so that $\mathbf{C}^{(2)}$ is the so-called standard additive coalescent (cf. 4, 1]). Next, for $1<\alpha<2$, we denote by $\sigma^{(\alpha)}=\left(\sigma^{(\alpha)}(t), t \geq 0\right)$ a standard spectrally positive stable Lévy process with index $\alpha$, that is $\sigma^{(\alpha)}$ has independent and stationary increments, no negative jumps, and

$$
\mathbb{E}\left(\exp \left(-\lambda \sigma^{(\alpha)}(t)\right)\right)=\exp \left(t \lambda^{\alpha}\right), \quad \text { for all } \lambda \geq 0 .
$$

We call standard stable loop ${ }^{1}$ of index $\alpha$ the process $\mathcal{B}^{(\alpha)}$ defined by

$$
\mathcal{B}^{(\alpha)}(x)=\sigma^{(\alpha)}(x)-x \sigma^{(\alpha)}(1), \quad \text { for } 0 \leq x \leq 1 .
$$

We finally write $\mathbf{C}^{(\alpha)}=\left(\mathbf{C}^{(\alpha)}(t),-\infty<t<\infty\right)$ for the eternal additive coalescent $\mathbf{C}$ constructed above when the bridge $B$ is the standard stable loop of index $\alpha$.

We are now able to state our main result.

\footnotetext{
${ }^{1}$ We call $\mathcal{B}^{(\alpha)}$ a loop and not a bridge to avoid a possible confusion: even though $\mathcal{B}^{(\alpha)}$ starts from 0 , ends at 0 and has exchangeable increments, it does not have the same law as the stable process $\sigma^{(\alpha)}$ conditioned on $\sigma^{(\alpha)}(1)=0$ !
} 
Theorem 1 The process $\left(\mathbf{X}^{(\varepsilon)}(t), 0 \leq t<T_{\varepsilon}\right)$ converges as $\varepsilon \downarrow 0$ in the sense of weak convergence of finite-dimensional distributions to some process $\mathbf{X}=(\mathbf{X}(t), 0 \leq t<\infty)$. The exponential time-changed process $\left(\mathbf{X}\left(e^{-t}\right),-\infty<t<\infty\right)$ is an eternal additive coalescent; more precisely:

(i) When $\alpha=2,\left(\mathbf{X}\left(e^{-t}\right),-\infty<t<\infty\right)$ is distributed as

$$
\left(\mathbf{C}^{(2)}\left(t+\frac{1}{2} \log \left(\mu_{2} / \mu_{1}\right)-\log \mu_{1}\right),-\infty<t<\infty\right) .
$$

(ii) When $1<\alpha<2,\left(\mathbf{X}\left(e^{-t}\right),-\infty<t<\infty\right)$ is distributed as

$$
\left(\mathbf{C}^{(\alpha)}\left(t+\frac{1}{\alpha} \log \left(\frac{\Gamma(2-\alpha) c}{(\alpha-1) \mu_{1}}\right)-\log \mu_{1}\right),-\infty<t<\infty\right) .
$$

It might be interesting to discuss further the role of the parameter $\alpha$ and the interpretation in terms of phase transition. As it was already mentioned, the renewal theorem entails than the number of drops of paint needed for the complete covering is $T_{\varepsilon} \sim 1 /\left(\varepsilon \mu_{1}\right)$, a quantity which is not sensitive to $\alpha$. It is easy to show that for every $a<1$, there are no macroscopic painted components when only $\left[a T_{\varepsilon}\right]$ drops of paint have fallen, so the phase transition (i.e. the number of drops which is needed for the appearance of macroscopic components) occurs for numbers close to $T_{\varepsilon}$. More precisely, the regime for the phase transition is of order $T_{\varepsilon}-\varepsilon^{-1 / \alpha}$; so the phase transition occurs closer to $T_{\varepsilon}$ when $\alpha$ is larger. We would like also to stress that one-dimensional distributions of the limiting additive coalescent process $\mathbf{X}$ depend on $\alpha$, but not its semigroup which is the same for all $\alpha \in(1,2]$. A heuristic explanation might be the following: the number of drops needed to complete the covering once the phase transition has occurred is too small (of order $\varepsilon^{-1 / \alpha}$ ) to observe significant differences in the dynamics of aggregation of macroscopic painted components.

Remark. Our model bears some similarity with another parking problem on the circle, where drops of paints fall uniformly on the circle and then are brushed clockwise, but where overlaps are now allowed (some points may be covered this way several times), call it the "random covering of an interval" problem. However, as showed in [6], this last model has very different asymptotics from those of the parking problem, as it turns out that the random covering of an interval is related to Kingman's coalescent rather than the additive coalescent. A shared feature is that the phase transition of the random covering problem appears also when the circle is almost completely covered, but for example the different fragments are ultimately finite in number rather than infinite.

We also mention yet another parking problem, first considered by Rényi (see [15, 9]). In can be formulated as follows: caravans with size $\varepsilon$ are placed on $\mathbb{T}$ (the original work rather considers $(0,1))$ one after another, but the locations $s_{i}$ where cars park are chosen uniformly among spaces that do not induce overlaps and splitting of caravans, i.e. so that the length of the arc from $s_{i}$ to $t_{i}$ is exactly $\varepsilon$. This is done until no uncovered sub-arc of $\mathbb{T}$ with size $\geq \varepsilon$ remains. This process does not involve coalescing blocks of cars, and one is rather interested in the properties of the random number of cars that are able to park.

The method in [8] relies on an encoding parking function which is shown to be asymptotically related to a function of standard Brownian bridge, and a representation of the standard additive coalescent due to Bertoin [4. Our approach to Theorem 1 is close in 
spirit to that of [8], and uses the representation of eternal additive coalescent that we presented above; we briefly sketch it here. First, we encode the process $\mathbf{X}^{(\varepsilon)}$ by a bridge with exchangeable increments in Sect. 2. In Sect. 3, we show that this bridge converges to some bridge with exchangeable increments that can be represented in terms of the standard Brownian bridge (for $\alpha=2$ ) or the standard stable loop (for $1<\alpha<2$ ). Theorem [1 then follows readily.

The rest of this work is organized as follows. In Section 2 we provide a representation of the painted components in terms of a bridge and its Vervaat's transform. The convergence of these bridges when $\varepsilon$ tends to 0 is established in Section 3, and that of the sequence of the sizes of the painted components in Section 4. Section 5 is devoted to a brief discussion of the analogous discrete setting (i.e. Knuth's parking for caravans), and finally some complements are presented in Section 6

\section{Bridge representation}

We develop a representation of the parking process with the help of bridges with exchangeable increments, which is crucial to our study.

Let us first give the proper definition the of sequence $\left(\varnothing=A_{0}, \ldots, A_{m}\right)$ of the Introduction. We identify the circle $\mathbb{T}$ with $[0,1)$ and write $p_{\mathbb{T}}: \mathbb{R} \rightarrow \mathbb{T}$ for the canonical projection. If $A$ is a measurable subset of $\mathbb{T}$ (identified with $[0,1)$ ), let $F_{A}$ be its repartition function defined by $F_{A}(x)=\operatorname{Leb}([0, x] \cap A)$ for $0 \leq x<1$, where Leb is Lebesgue measure. Also, extend $F_{A}$ on the whole real line with the formula $F_{A}(x+1)=F_{A}(x)+F_{A}(1-)$. Given $A_{i}$ for some $0 \leq i \leq m-1$, let

$$
t_{i+1}=\inf \left\{x \geq s_{i+1}: F_{A_{i}}(x)+p_{i+1}-\left(x-s_{i+1}\right) \leq F_{A_{i}}\left(s_{i+1}\right)\right\} .
$$

Notice that the arc $p_{\mathbb{T}}\left(\left(s_{i+1}, t_{i+1}\right)\right)$ oriented clockwise from $s_{i+1}$ to $p_{\mathbb{T}}\left(t_{i+1}\right)$ has length $t_{i+1}-s_{i+1} \geq p_{i+1}$. Then let $A_{i+1}$ be the interior of the closure of $p_{\mathbb{T}}\left(\left(s_{i+1}, t_{i+1}\right)\right) \cup A_{i}$. The point in taking the closure and then the interior is that we consider that two painted connected components of $\mathbb{T}$ that are at distance 0 constitute in fact a single painted connected component.

Define

$$
h_{i+1}^{\mathbf{p}}(x)=F_{A_{i}}(x)-F_{A_{i}}\left(s_{i+1}\right)+p_{i+1}-\left(x-s_{i+1}\right) \quad s_{i+1} \leq x \leq t_{i+1},
$$

and $h_{i+1}^{\mathbf{p}}(x)=0$ in $\left[t_{i+1}, s_{i+1}+1\right.$ ), so $h_{i}^{\mathbf{p}}$ is a càdlàg function (right-continuous with leftlimits) on $\left[s_{i+1}, s_{i+1}+1\right)$. Consider it as a function on $\mathbb{T}$ by letting $h_{i+1}^{\mathbf{p}}(x)=h_{i+1}^{\mathbf{p}}(y)$ where $y$ is the element of $\left[s_{i+1}, s_{i+1}+1\right) \cap p_{\mathbb{T}}^{-1}(x)$. The quantity $h_{i+1}^{\mathbf{p}}(x)$ can be thought of as the quantity of cars of the $i+1$-th caravan that try to park at $x$. See Figure 2

We consider the profile

$$
H_{i}^{\mathbf{p}}=\sum_{j=1}^{i} h_{j}^{\mathbf{p}}
$$

of the parking at step $0 \leq i \leq m$, so $H_{i}^{\mathbf{p}}(x)$ is the total quantity of cars that have tried (successfully or not) to park at $x$ (with the convention that $H_{i}^{\mathbf{p}}(1)=H_{i}^{\mathbf{p}}(0)$ ) before the $i+1$-th caravan has arrived. 


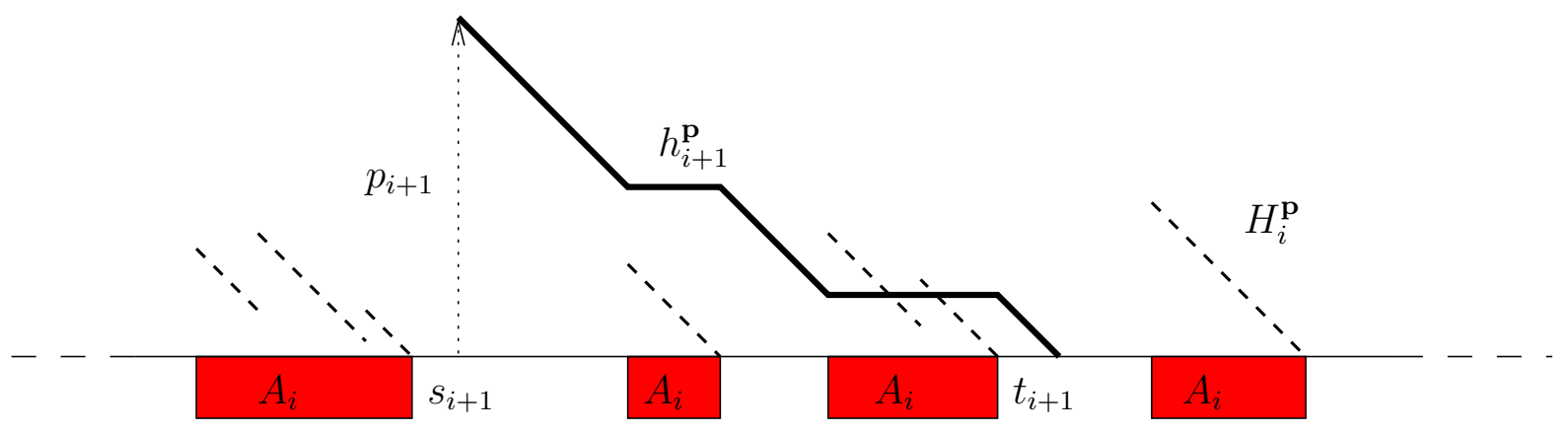

Figure 2: The function $h_{i+1}^{\mathbf{p}}$ (thick line) corresponding to the $i+1$-th caravan of Figure 1 The blocks of $A_{i}$ are represented under the axis, and the dashed lines represent the profile $H_{i}^{\mathrm{p}}$ (it gives more information than $A_{i}$ alone). The bracket under the figure indicates how $A_{i+1}$ is obtained by formation of a new block comprising the blocks of $A_{i}$ between $s_{i+1}$ and $t_{i+1}$

Lemma 1 For $1 \leq i \leq m$,

(i) the set $A_{i}$ is the interior of the support of $H_{i}^{\mathrm{p}}$.

(ii) $H_{i}^{\mathbf{p}}\left(t_{i}-\right)=0$.

(iii) $H_{i}^{\mathbf{p}}$ jumps at times $s_{1}, \ldots, s_{i}$ with respective jump magnitudes $p_{1}, \ldots, p_{i}$, and has a drift with slope -1 on its support. That is, if $\left[v, v^{\prime}\right] \subseteq \operatorname{supp}\left(H_{i}^{\mathbf{p}}\right)$,

$$
H_{i}^{\mathbf{p}}(x+v)=H_{i}^{\mathbf{p}}(v-)-x+\sum_{j=1}^{i} p_{j} \mathbb{1}_{\left\{v \leq s_{j} \leq v+x\right\}} \quad 0 \leq x \leq v^{\prime}-v .
$$

Proof. Properties (i) and (iii) are easily shown using a recursion on $i$ and splitting the behavior of $h_{i}^{\mathbf{p}}$ on $A_{i-1}$ and $A_{i} \backslash A_{i-1}$. We give some details for (ii). For $i \geq 1$, notice that by definition $t_{i}$ cannot be a point of increase of $F_{A_{i-1}}$, i.e. a point such that $F_{A_{i-1}}\left(t_{i}-\varepsilon\right)<F_{A_{i-1}}\left(t_{i}\right)<F_{A_{i-1}}\left(t_{i}+\varepsilon\right)$ for every $\varepsilon>0$. Therefore, $t_{i} \notin A_{i-1}$ and $h_{j}^{\mathbf{p}}\left(t_{i}\right)=h_{j}^{\mathbf{p}}\left(t_{i}-\right)=0$ for $j<i$. Since it follows by continuity of $F_{A_{i-1}}$ that $h_{i}^{\mathbf{p}}\left(t_{i}-\right)=0$, (ii) is proved.

Consider the bridge function:

$$
b_{i}^{\mathbf{p}}(x)=-x+\sum_{j=1}^{i} p_{j} \mathbb{1}_{\left\{x \geq s_{j}\right\}} \quad 0 \leq x<1,
$$

which starts from $b_{i}^{\mathbf{p}}(0)=0$ and ends at $b_{i}^{\mathbf{p}}(1-)=p_{1}+\ldots+p_{i}-1$. We extend $b_{i}^{\mathbf{p}}$ to a function on $\mathbb{R}$ by setting $b_{i}^{\mathbf{p}}(x+1)=b_{i}^{\mathbf{p}}(x)+b_{i}^{\mathbf{p}}(1-)$. For any $v \in[0,1)$, it is easily seen using (iii) in Lemma 1 that

$$
H_{i}^{\mathbf{p}}(x+v)=H_{i}^{\mathbf{p}}(v-)+b_{i}^{\mathbf{p}}(x+v)-\left(0 \wedge \inf _{u \in[v, v+x]}\left(H_{i}^{\mathbf{p}}(v-)+b_{i}^{\mathbf{p}}(u)\right)\right) .
$$

Suppose $v$ is such that $H_{m}^{\mathbf{p}}(v-)=0$ (here $\left.H_{m}^{\mathbf{p}}(0-)=H_{m}^{\mathbf{p}}(1-)\right)$, call such a number a last empty spot. By (ii), Lemma 1 the set of last empty spots is not empty since it contains 
$t_{m}$. On the other hand, by (i) in the same lemma, the support of $H_{m}^{\mathrm{p}}$ is the closure of $A_{m}$ which has measure 1 , hence it is $\mathbb{T}$. By (iii), we conclude by letting $v=0, v^{\prime} \uparrow 1$ that $H_{m}^{\mathbf{p}}(x)=H_{m}^{\mathbf{p}}(0)+b_{m}^{\mathbf{p}}(x)$ for $0 \leq x<1$, so for $x=t_{m}-, H_{m}^{\mathbf{p}}(0)=-b_{m}^{\mathbf{p}}\left(t_{m}-\right)=-\inf b_{m}^{\mathbf{p}}$ necessarily since $H_{m}^{\mathrm{p}}$ is non-negative. This implies that the last empty spots are those $v$ 's such that $b_{m}^{\mathbf{p}}(v-)=\inf b_{m}^{\mathbf{p}}$. We choose one of them by letting

$$
V=\inf \left\{x \in[0,1]: b_{m}^{\mathbf{p}}(x-)=\inf _{u \in[0,1]} b_{m}^{\mathbf{p}}(u)\right\}
$$

the first location when the infimum of $b_{m}^{\mathbf{p}}$ is reached. We have proved

Lemma 2 For any $0 \leq x<1,1 \leq i \leq m$,

$$
H_{i}^{\mathbf{p}}(x+V)=b_{i}^{\mathbf{p}}(x+V)-\inf _{u \in[V, V+x]} b_{i}^{\mathbf{p}}(u) .
$$

Recall that we are interested in $\Lambda^{\mathrm{p}}(i)$, the ranked sequence of the lengths of the interval components of $A_{i}$, where $A_{i}$ can be viewed as the painted portion of the circle after $i$ drops of paint have fallen, or the set of occupied spots after the $i$-th caravan has arrived. Lemma [1(i) enables us to identify $A_{i}$ as the interior of support of the function $H_{i}^{\mathrm{p}}$, and since the Lebesgue measure of the interval components of the interior of the support of $H_{i}^{\mathbf{p}}$ is not affected by a cyclic shift, we record the following simple identification

Lemma 3 For every $i=1, \ldots, m, \Lambda^{\mathbf{p}}(i)$ coincides with the ranked lengths of the intervals of constancy of the function

$$
x \longmapsto \inf _{u \in[V, V+x]} b_{i}^{\mathbf{p}}(u), \quad x \in[0,1] .
$$

\section{Convergence of bridges}

We now consider a rescaled randomized version of the bridges introduced above. Let $B^{(\varepsilon)}=\varepsilon^{-1+1 / \alpha} b_{m}^{\mathbf{p}}$, where $b_{m}^{\mathbf{p}}$ is obtained as above with data $m=T_{\varepsilon}, p_{i}=\varepsilon \ell_{i}^{*}, s_{i}=U_{i}$, and these quantities are introduced in the Introduction. So for $0 \leq x \leq 1$

$$
B^{(\varepsilon)}(x)=-\varepsilon^{-1+1 / \alpha} x+\sum_{i=1}^{T_{\varepsilon}} \varepsilon^{1 / \alpha} \ell_{i}^{*} \mathbb{1}_{\left\{x \geq U_{i}\right\}}=\varepsilon^{1 / \alpha} \sum_{i=1}^{T_{\varepsilon}} \ell_{i}^{*}\left(\mathbb{1}_{\left\{x \geq U_{i}\right\}}-x\right),
$$

because $\ell_{1}^{*}+\ldots+\ell_{T_{\varepsilon}}^{*}=1 / \varepsilon$. Recall that $\mathcal{B}^{(2)}$ denotes the standard Brownian bridge, and $\mathcal{B}^{(\alpha)}$ the standard stable loop with index $\alpha$ as defined in (2).

Lemma $4 A s \varepsilon \downarrow 0$, the bridge $B^{(\varepsilon)}$ converges weakly on the space $\mathbb{D}$ of càdlàg paths endowed with Skorokhod's topology, to a bridge with exchangeable increments $B=(B(x), 0 \leq$ $x \leq 1)$. More precisely:

(i) If $\alpha=2$ then $B$ is distributed as $\sqrt{\mu_{2} / \mu_{1}} \mathcal{B}^{(2)}$.

(ii) If $\alpha \in(1,2)$, then $B$ is distributed as

$$
\left(\frac{\Gamma(2-\alpha) c}{(\alpha-1) \mu_{1}}\right)^{\frac{1}{\alpha}} \mathcal{B}^{(\alpha)}
$$


The proof of Lemma 4(ii) will use the following well-known representation:

$$
\left(\frac{\Gamma(2-\alpha) c}{(\alpha-1) \mu_{1}}\right)^{\frac{1}{\alpha}} \mathcal{B}^{(\alpha)}(x)=\sum_{i=1}^{\infty} \Delta_{i}\left(\mathbb{1}_{\left\{x \geq U_{i}\right\}}-x\right), \quad 0 \leq x \leq 1,
$$

where $\left(U_{i}, i \geq 1\right)$ is a sequence of i.i.d. uniform $(0,1)$ r.v.'s, $\left(\Delta_{i}, i \geq 1\right)$ is the ranked sequence of the atoms of a Poisson measure on $(0, \infty)$ with intensity $\alpha c \mu_{1}^{-1} x^{-1-\alpha} \mathrm{d} x$, and these two sequences are independent. More precisely, the series in the right-hand side does not converge absolutely, but is taken in the sense

$$
\sum_{i=1}^{\infty} \Delta_{i}\left(\mathbb{1}_{\left\{x \geq U_{i}\right\}}-x\right)=\lim _{n \rightarrow \infty} \sum_{i=1}^{n} \Delta_{i}\left(\mathbb{1}_{\left\{x \geq U_{i}\right\}}-x\right),
$$

where the limit is uniform in the variable $x$, a.s. This representation follows immediately from the celebrated Lévy-Itô decomposition, specified for the stable process $\sigma^{(\alpha)}$, as the process of the jumps of the latter is a Poisson point process on $\mathbb{R}_{+}$with intensity $\frac{\alpha(\alpha-1)}{\Gamma(2-\alpha)} x^{-1-\alpha} \mathrm{d} x$. See also Kallenberg [11].

Proof. Following Kallenberg [11], we represent the jump sizes of the bridge $B^{(\varepsilon)}$ by the random point measure

$$
\psi_{\varepsilon}=\sum_{i=1}^{T_{\varepsilon}}\left(\varepsilon^{1 / \alpha} \ell_{i}^{*}\right)^{2} \delta_{\varepsilon^{1 / \alpha} \ell_{i}^{*}}
$$

By Theorem 2.3 in [11, we have to show:

$$
\text { if } \alpha=2 \text {, then } \psi_{\varepsilon} \rightarrow\left(\mu_{2} / \mu_{1}\right) \delta_{0},
$$

and

$$
\text { if } \alpha<2 \text {, then } \psi_{\varepsilon} \rightarrow \psi:=\sum_{i=1}^{\infty} \Delta_{i}^{2} \delta_{\Delta_{i}}
$$

where the convergence is in law with respect to the weak topology on measures on $[0, \infty)$, and in (5),$\left(\Delta_{i}, i \geq 1\right)$ is the ranked sequence of the atoms of a Poisson measure on $(0, \infty)$ with intensity $\alpha c \mu_{1}^{-1} x^{-1-\alpha} \mathrm{d} x$.

Case (i) is easier to treat. Indeed, notice that the total mass of $\psi_{\varepsilon}$ is

$$
\psi_{\varepsilon}\left(\mathbb{R}_{+}\right)=\varepsilon \sum_{i=1}^{T_{\varepsilon}}\left(\ell_{i}^{*}\right)^{2}=\varepsilon T_{\varepsilon} \frac{\left(\ell_{T_{\varepsilon}}^{*}\right)^{2}+\sum_{i=1}^{T_{\varepsilon}-1} \ell_{i}^{2}}{T_{\varepsilon}}
$$

Since $\ell_{i}^{*} \leq \ell_{i}$, the law of large numbers gives $\psi_{\varepsilon}\left(\mathbb{R}_{+}\right) \rightarrow \mu_{2} / \mu_{1}$.

Now let

$$
m_{\varepsilon}^{*}:=\sqrt{\varepsilon} \max _{1 \leq i \leq T_{\varepsilon}} \ell_{i}^{*} \quad \text { and } \quad M_{n}:=\sqrt{\varepsilon} \max _{1 \leq i \leq n} \ell_{i},
$$

so to prove (44), it suffices to show that $m_{\varepsilon}^{*} \rightarrow 0$ in probability. Notice that $m_{\varepsilon}^{*} \leq M_{T_{\varepsilon}}$.

Let $\eta>0$ and $K>\mu_{1}^{-1}$. Then

$$
\begin{aligned}
\mathbb{P}\left(m_{\varepsilon}^{*}>\eta\right) & =\mathbb{P}\left(m_{\varepsilon}^{*}>\eta, T_{\varepsilon} \leq K \varepsilon^{-1}\right)+\mathbb{P}\left(m_{\varepsilon}^{*}>\eta, T_{\varepsilon}>K \varepsilon^{-1}\right) \\
& \leq \mathbb{P}\left(M_{\left\lfloor K \varepsilon^{-1}\right\rfloor}>\eta\right)+\mathbb{P}\left(T_{\varepsilon}>K \varepsilon^{-1}\right) .
\end{aligned}
$$


The second term converges to 0 since $\varepsilon \mu_{1} T_{\varepsilon} \rightarrow 1$ a.s. For the first term, notice that

$$
\mathbb{P}\left(M_{\left\lfloor K \varepsilon^{-1}\right\rfloor} \leq \eta\right)=(1-\mathbb{P}(\ell>\eta / \sqrt{\varepsilon}))^{\left\lfloor K \varepsilon^{-1}\right\rfloor} .
$$

Taking logarithms and checking that $\varepsilon^{-1} \mathbb{P}\left(\ell^{2}>\eta^{2} / \varepsilon\right) \rightarrow 0$ as $\varepsilon \downarrow 0$ (which holds since $\left.\mathbb{E}\left[\ell^{2}\right]<\infty\right)$, we finally obtain that $\mathbb{P}\left(M_{\left\lfloor K \varepsilon^{-1}\right\rfloor} \leq \eta\right) \rightarrow 1$. This completes the proof of (44).

Now we turn our attention to (5). It suffices to show that for every function $f$ : $[0, \infty) \rightarrow[0, \infty)$, say of class $\mathcal{C}^{1}$ with bounded derivative

$$
\lim _{\varepsilon \rightarrow 0} \mathbb{E}\left(\exp \left(-\left\langle\psi_{\varepsilon}, f\right\rangle\right)\right)=\mathbb{E}(\exp (-\langle\psi, f\rangle)) ;
$$

see for instance Section II.3 in Le Gall [12]. In this direction, recall from the classical formula for Poisson random measures that

$$
\mathbb{E}(\exp (-\langle\psi, f\rangle))=\frac{\alpha c}{\mu_{1}} \int_{0}^{\infty}\left(1-\exp \left(-y^{2} f(y)\right)\right) y^{-1-\alpha} d y
$$

To start with, we observe from the renewal theorem that $\varepsilon^{1 / \alpha} \ell_{T_{\varepsilon}}^{*}$ converges to 0 in probability as $\varepsilon \rightarrow 0$, so in (6), we may replace $\psi_{\varepsilon}$ by

$$
\psi_{\varepsilon}^{\prime}=\sum_{i=1}^{T_{\varepsilon}-1}\left(\varepsilon^{1 / \alpha} \ell_{i}\right)^{2} \delta_{\varepsilon^{1 / \alpha} \ell_{i}} .
$$

Next, for every $a \geq 0$, we consider the random measure

$$
\psi_{\varepsilon, a}=\sum_{i=1}^{a / \varepsilon}\left(\varepsilon^{1 / \alpha} \ell_{i}\right)^{2} \delta_{\varepsilon^{1 / \alpha} \ell_{i}} .
$$

Again, by the (elementary) renewal theorem, $\varepsilon T_{\varepsilon} \rightarrow \mu_{1}^{-1}$ in probability, so for every $\eta>0$, the event

$$
\left\langle\psi_{\varepsilon, \mu_{1}^{-1}-\eta}, f\right\rangle \leq\left\langle\psi_{\varepsilon}^{\prime}, f\right\rangle \leq\left\langle\psi_{\varepsilon, \mu_{1}^{-1}+\eta}, f\right\rangle
$$

has a probability which tends to 1 as $\varepsilon \rightarrow 0$.

Now

$$
\mathbb{E}\left(\exp \left(-\left\langle\psi_{\varepsilon, a}, f\right\rangle\right)\right)=\mathbb{E}\left(\exp \left(-f\left(\varepsilon^{1 / \alpha} \ell\right)\left(\varepsilon^{1 / \alpha} \ell\right)^{2}\right)\right)^{a / \varepsilon} .
$$

Taking logarithms, we have to estimate

$$
\begin{aligned}
& \frac{a}{\varepsilon} \mathbb{E}\left(1-\exp \left(-f\left(\varepsilon^{1 / \alpha} \ell\right)\left(\varepsilon^{1 / \alpha} \ell\right)^{2}\right)\right) \\
= & \frac{a}{\varepsilon} \int_{0}^{\infty} \varepsilon^{2 / \alpha}\left(2 x f\left(\varepsilon^{1 / \alpha} x\right)+\varepsilon^{1 / \alpha} x^{2} f^{\prime}\left(\varepsilon^{1 / \alpha} x\right)\right) \exp \left(-\left(\varepsilon^{1 / \alpha} x\right)^{2} f\left(\varepsilon^{1 / \alpha} x\right)\right) \mathbb{P}(\ell>x) d x \\
= & \frac{a}{\varepsilon} \int_{0}^{\infty}\left(2 y f(y)+y^{2} f^{\prime}(y)\right) \exp \left(-y^{2} f(y)\right) \mathbb{P}\left(\ell>y / \varepsilon^{1 / \alpha}\right) d x .
\end{aligned}
$$

By (11) and dominated convergence, we see that the preceding quantity converges as $\varepsilon \rightarrow 0$ towards

$$
a c \int_{0}^{\infty}\left(2 y f(y)+y^{2} f^{\prime}(y)\right) \exp \left(-y^{2} f(y)\right) y^{-\alpha} d x=\alpha a c \int_{0}^{\infty}\left(1-\exp \left(-y^{2} f(y)\right)\right) y^{-1-\alpha} d x .
$$

Taking $a=\mu_{1}^{-1} \pm \eta$, using (77) and letting $\eta$ tend to 0 , we see that (6) holds, which completes the proof of the statement. 


\section{Convergence of $\mathrm{X}^{(\varepsilon)}$}

In this section, we deduce Theorem 1 from Lemmas 34. Recall the definition of the bridge $b_{i}^{\mathbf{p}}$ in Section 2, For $i \leq T_{\varepsilon}$, let $B_{i}^{(\varepsilon)}$ be the bridge $\varepsilon^{-1+1 / \alpha} b_{i}^{\mathbf{p}}$ with data $p_{j}=\varepsilon \ell_{j}^{*}, s_{j}=U_{j}$, so $B_{T_{\varepsilon}}^{(\varepsilon)}=B^{(\varepsilon)}$. Let also $V_{\varepsilon}$ be the left-most location of the infimum of $B^{(\varepsilon)}$, and

$$
\mathbf{V} B^{(\varepsilon)}(x)=B^{(\varepsilon)}\left(x+V_{\varepsilon}\right)-\inf B^{(\varepsilon)}, \quad 0 \leq x \leq 1
$$

the Vervaat transform of $B^{(\varepsilon)}$. By Lemma 3, $\mathbf{X}^{(\varepsilon)}(t)=\Lambda^{\mathbf{p}}\left(T_{\varepsilon}-\left\lfloor t \varepsilon^{-1 / \alpha}\right\rfloor\right)$ coincides with the ranked sequence of lengths of constancy intervals of the infimum process of

$$
B_{T_{\varepsilon}-\left\lfloor t \varepsilon^{-1 / \alpha}\right\rfloor}^{(\varepsilon)}\left(x+V_{\varepsilon}\right)-\inf B^{(\varepsilon)}, \quad 0 \leq x \leq 1,
$$

where the constant $-\inf B^{(\varepsilon)}$ has no effect and is added for future considerations.

Lemma 5 For every $t \geq 0$, the difference

$$
B^{(\varepsilon)}(x)-B_{T_{\varepsilon}-\left\lfloor t \varepsilon^{-1 / \alpha}\right\rfloor}^{(\varepsilon)}(x)=\varepsilon^{1 / \alpha} \sum_{j=0}^{\left\lfloor t \varepsilon^{-1 / \alpha}-1\right\rfloor} \ell_{T_{\varepsilon}-i}^{*} \mathbb{1}_{\left\{x \geq U_{T_{\varepsilon}-i}\right\}} \quad 0 \leq x \leq 1
$$

converges in probability for the uniform norm to the pure drift $x \mapsto t \mu_{1} x$ as $\varepsilon \downarrow 0$.

Proof. Recall from the renewal theorem that $\varepsilon^{1 / \alpha} \ell_{T_{\varepsilon}} \rightarrow 0$ in probability as $\varepsilon \downarrow 0$. Therefore, we might start the sum appearing in the statement from $j=1$. Now, the sequences $\left(\ell_{1}, \ldots, \ell_{T_{\varepsilon}-1}\right)$ and $\left(\ell_{T_{\varepsilon}-1}, \ldots, \ell_{1}\right)$ have the same distribution. Up to doing the substitution, Lemma 5 for fixed $s$ is therefore a simple application of the strong law of large numbers. The conclusion is obtained by standard monotonicity arguments.

As a consequence of Lemmas 4 , 5 , and the fact that $s \mapsto t \mu_{1} s$ is continuous, the process

$$
B_{T_{\varepsilon}-\left\lfloor t \varepsilon^{-1 / \alpha}\right\rfloor}^{(\varepsilon)}\left(x+V_{\varepsilon}\right)-\inf B^{(\varepsilon)}=\mathbf{V} B^{(\varepsilon)}(x)-\left(B^{(\varepsilon)}\left(x+V_{\varepsilon}\right)-B_{T_{\varepsilon}-\left\lfloor t \varepsilon^{-1 / \alpha}\right\rfloor}^{(\varepsilon)}\left(x+V_{\varepsilon}\right)\right)
$$

converges in the Skorokhod space to

$$
\mathcal{E}^{\left(t \mu_{1}\right)}=\left(\mathcal{E}(x)-t \mu_{1} x, 0 \leq x \leq 1\right)
$$

where

$$
\mathcal{E}(x)=B(x+V)-\inf B, \quad 0 \leq x \leq 1
$$

is the Vervaat transform of the limiting bridge $B$ which appears in Lemma 4, $V$ being the a.s. unique location of its infimum. Now letting $\underline{\mathcal{E}}^{(t)}$ be the infimum process of $\mathcal{E}^{(t)}$ and $\mathbf{F}(t)$ be the decreasing sequence of lengths of constancy intervals of $\underline{\mathcal{E}}^{(t)}$, we have

Proposition 1 The process $\left(\mathbf{X}^{(\varepsilon)}(t), t \geq 0\right)$ converges to $\left(\mathbf{F}\left(\mu_{1} t\right), t \geq 0\right)$ in the sense of weak convergence of finite-dimensional marginals. 
Proof. The technical point is that Skorokhod convergence of $B_{T_{\varepsilon}-\left\lfloor t \varepsilon^{-1 / \alpha}\right\rfloor}^{(\varepsilon)}\left(x+V_{\varepsilon}\right)-\inf B^{(\varepsilon)}$ to $\mathcal{E}^{\left(t \mu_{1}\right)}$, though it does imply convergence of respective infimum processes, does not a priori imply that of the ranked sequence of lengths of constancy intervals of these processes. However, this convergence does hold because for every $t \geq 0$, if $(a, b)$ is such a constancy interval, then $\mathcal{E}^{\left(t \mu_{1}\right)}(x)>\mathcal{E}^{\left(t \mu_{1}\right)}(a)$ for $x \in(a, b)$, a.s. See e.g. Lemmas 4 and 7 in [5].

This proposition proves Theorem 1] Indeed, recall from Lemma 4 that $B=c_{\alpha} \mathcal{B}_{\alpha}$, where $c_{2}=\sqrt{\mu_{2} / \mu_{1}}$ and for $1<\alpha<2$

$$
c_{\alpha}=\left(\frac{\Gamma(2-\alpha) c}{(\alpha-1) \mu_{1}}\right)^{\frac{1}{\alpha}} .
$$

Then plainly, $\mathbf{F}\left(e^{-t}\right)=\mathbf{C}^{(\alpha)}\left(t+\log c_{\alpha}\right)$, and hence the limiting process $\mathbf{X}\left(e^{-t}\right)$ is distributed as $\mathbf{F}\left(\mu_{1} e^{-t}\right)=\mathbf{C}^{(\alpha)}\left(t+\log c_{\alpha}-\log \mu_{1}\right)$.

\section{Related results for a discrete problem}

In situations involving parking problems, it may be more natural to consider discrete parking lots, i.e. $\mathbb{Z} / n \mathbb{Z}$ instead of the unit circle, and caravans with integer sizes, e.g. as in Knuth's original parking problem. Each caravan chooses a random spot, uniform on $\mathbb{Z} / n \mathbb{Z}$, and tries to park at that spot. Studying the frequencies of blocks of cars fits with our general framework by taking $\ell$ with integer values, $\varepsilon=1 / n$ and $s_{i}=\left\lfloor n U_{i}\right\rfloor / n$. Rename by $T_{n}$ the former quantity $T_{\varepsilon}$ (the number of caravans). Let

$$
\begin{aligned}
& \widetilde{B}^{(n)}(x)=n^{1 / \alpha} \sum_{i=1}^{T_{n}}\left(\frac{\ell_{i}^{*}}{n} \mathbb{1}_{\left\{x \geq\left\lfloor n U_{i}\right\rfloor / n\right\}}-x\right) \quad 0 \leq x \leq 1 \\
& B^{(n)}(x)=n^{1 / \alpha} \sum_{i=1}^{T_{n}}\left(\frac{\ell_{i}^{*}}{n} \mathbb{1}_{\left\{x \geq U_{i}\right\}}-x\right) \quad 0 \leq x \leq 1,
\end{aligned}
$$

so $B^{(n)}$ would be $B^{(1 / n)}$ in the notation above. The analog of Lemma 5 is still true when replacing $B^{(n)}$ by $\widetilde{B}^{(n)}$, without essential change in the proof. Thus to obtain the very same conclusions as in the preceding sections, it suffices to check a result similar to Lemma 4 Namely, we must prove that $\widetilde{B}^{(n)} \rightarrow B$ in the Skorokhod space as $n \rightarrow \infty$. Now it is easy to check that a.s., $\left|\widetilde{B}^{(n)}(x)-B^{(n)}(\lceil n x-\rceil / n)\right| \leq n^{1 / \alpha} / n$ for every $n \geq 1, x \in[0,1]$, because no $U_{i}$ is rational a.s. Therefore, it suffices to check that $B^{(n)}(\lceil n \cdot+\rceil / n)$ converges to $B$ in distribution for the Skorokhod topology on $\mathbb{D}$. Up to using Skorokhod's representation theorem, this is done by taking $f_{n}=B^{(n)}$ and $\kappa_{n}(x)=\lceil n x+\rceil / n$ in the next lemma.

Lemma 6 Let $\left(f_{n}, n \geq 1\right)$ be a sequence of functions converging in $\mathbb{D}$ to $f$. For $n \in \mathbb{N}$ let also $\kappa_{n}$ be a right-continuous non-decreasing function (not necessarily bijective) from $[0,1]$ to $[0,1]$, such that the sequence $\left(\kappa_{n}\right)$ converges to the identity function uniformly on $[0,1]$. Then $f_{n} \circ \kappa_{n} \rightarrow f$ in $\mathbb{D}$.

Proof. First consider the case $f_{n}=f$ for every $n$. Fix $\varepsilon>0$. Let $\kappa_{n}^{-1}$ be the rightcontinuous inverse of $\kappa_{n}$ defined by

$$
\kappa_{n}^{-1}(x)=\inf \left\{y \in[0,1]: \kappa_{n}(y)>x\right\} .
$$


It is easy to prove that $\kappa_{n}\left(\kappa_{n}^{-1}(x)-\right) \leq x \leq \kappa_{n}\left(\kappa_{n}^{-1}(x)\right)$ for every $x$. Since $f$ is càdlàg, one may find $0=x_{0}<x_{1}<\ldots<x_{k}=1$ such that the oscillation $\omega\left(f,\left[x_{i}, x_{i+1}\right)\right)<\varepsilon$ for $0 \leq i \leq k-1$, where

$$
\omega(f, A)=\sup _{x, y \in A}|f(x)-f(y)| .
$$

Since $\kappa_{n}$ approaches the identity, for $n$ large we may assume $\kappa_{n}\left(\kappa_{n}^{-1}\left(x_{i}\right)\right)<\kappa_{n}\left(\kappa_{n}^{-1}\left(x_{i+1}\right)-\right)$ for $0 \leq i \leq k-1$. Define a time-change $\lambda_{n}$ (i.e. an increasing bijection between $[0,1]$ and $[0,1])$ by interpolating linearly between the points $(0,0),\left(\kappa_{n}^{-1}\left(x_{i}\right), x_{i}\right), 1 \leq i \leq k-1,(1,1)$.

Now let $x \in[0,1]$. Suppose $\kappa_{n}^{-1}\left(x_{i}\right) \leq x<\kappa_{n}^{-1}\left(x_{i+1}\right)$ for some $0 \leq i \leq k-1$, and notice that $x_{i} \leq \kappa_{n}\left(\kappa_{n}^{-1}\left(x_{i}\right)\right) \leq \kappa_{n}(x)<\kappa_{n}\left(\kappa_{n}^{-1}\left(x_{i+1}\right)-\right) \leq x_{i+1}$. Therefore, $\kappa_{n}(x)$ belongs to $\left[x_{i}, x_{i+1}\right)$ as well as $\lambda_{n}(x)$ by definition of $\lambda_{n}$, and

$$
\left|f\left(\kappa_{n}(x)\right)-f\left(\lambda_{n}(x)\right)\right| \leq \omega\left(f,\left[x_{i}, x_{i+1}\right)\right) \leq \varepsilon .
$$

Else, one must have $x<\kappa_{n}^{-1}(0)$ or $x \geq \kappa_{n}^{-1}(1)$, and the result is similar. Finally, doing the same reasoning for $\varepsilon=\varepsilon_{n}$ converging to 0 slowly enough gives the existence of some timechanges $\lambda_{n}$ converging to the identity uniformly such that $\sup _{x \in[0,1]}\left|f\left(\kappa_{n}(x)\right)-f\left(\lambda_{n}(x)\right)\right| \leq$ $2 \varepsilon_{n}$, hence giving convergence of $f \circ \kappa_{n}$ to $f$ in the Skorokhod space.

In the general case, for every $n \geq 0$ let $\lambda_{n}$ be a time-change such that $\lambda_{n}$ converges to the identity as $n \rightarrow \infty$ and $f_{n} \circ \lambda_{n}$ converges to $f$ uniformly. Take $\kappa_{n}^{\prime}=\lambda_{n}^{-1} \circ \kappa_{n}$. Then $f_{n} \circ \kappa_{n}-f \circ \kappa_{n}^{\prime} \rightarrow 0$ uniformly, so it suffices to show that $f \circ \kappa_{n}^{\prime} \rightarrow f$ in $\mathbb{D}$, which is done by the former discussion.

In particular, we recover and extend a certain number of results from [8].

\section{Complements}

In this section, we would like to provide some information on the eternal additive coalescents $\mathbf{C}^{(\alpha)}$ for $1<\alpha<2$, which appear in Theorem 1 .

\subsection{Mixture of extremes}

To start with, we should like to specify the representation of $\mathbf{C}^{(\alpha)}$ as a mixture of socalled extreme eternal additive coalescents ([3], 5]). In this direction, let us first consider a sequence $\boldsymbol{\theta}=\left(\theta_{0}, \theta_{1}, \theta_{2}, \ldots\right)$ of non-negative numbers satisfying $\sum_{i \geq 0} \theta_{i}^{2}=1$ and

$$
\text { either } \theta_{0}>0 \quad \text { or } \quad \sum_{i \geq 0} \theta_{i}=\infty \text {. }
$$

Following Kallenberg [1] we associate to $\boldsymbol{\theta}$ a bridge with exchangeable increments

$$
B^{\theta}(x)=\theta_{0} \beta(x)+\sum_{i \geq 1} \theta_{i}\left(\mathbb{1}_{\left\{x \geq U_{i}\right\}}-x\right) \quad 0 \leq x \leq 1
$$

where $\left(U_{i}, i \geq 1\right)$ denotes a sequence of iid uniform variables and $\beta$ is an independent standard Brownian bridge. We write $\mathbf{C}^{\theta}$ for the eternal additive coalescent associated to the bridge $B=B^{\theta}$ as explained in the Introduction and call such $\mathbf{C}^{\theta}$ extreme.

According to [3. Theorem 15], every eternal version of the additive coalescent $\mathbf{C}$ can be obtained as a mixing of shifted versions of extreme eternal additive coalescents $\mathbf{C}^{\theta}$, i.e. 
$\mathbf{C}$ can be expressed in the form $\left(\mathbf{C}^{\theta^{*}}\left(t-t^{*}\right), t \in \mathbb{R}\right)$ with $\boldsymbol{\theta}^{*}, t^{*}$ random. Equivalently, $\mathbf{C}$ can be viewed as the eternal additive coalescent constructed in the Introduction from the bridge with exchangeable increments $B=e^{t^{*}} B^{\theta^{*}}$. As observed by Aldous and Pitman [3], the mixing variables $\boldsymbol{\theta}^{*}, t^{*}$ can be recovered from the initial behavior of $\mathbf{C}$ :

$$
e^{t^{*}} \theta_{i}^{*}=\lim _{t \rightarrow-\infty} e^{-t} \mathbf{C}_{i}(t) \text { and } e^{2 t^{*}}=\lim _{t \rightarrow-\infty} e^{-2 t} \sum_{i=1}^{\infty} \mathbf{C}_{i}^{2}(t) .
$$

In the case of the standard stable loop $\mathcal{B}^{(\alpha)}$ with $1<\alpha<2$, recall from the Lévy-Itô decomposition that $\theta_{0}^{*}=0$ and $\left(e^{t^{*}} \theta_{1}^{*}, e^{t^{*}} \theta_{2}^{*}, \ldots\right)=\left(\Delta_{1}, \Delta_{2}, \ldots\right)$ is the ranked sequence of the atoms of a Poisson random measure on $(0, \infty)$ with intensity $\frac{\alpha(\alpha-1)}{\Gamma(2-\alpha)} x^{-1-\alpha} d x$. In particular,

$$
e^{2 t^{*}}=\sum_{i=1}^{\infty} \Delta_{i}^{2}
$$

has the law of a (positive) stable variable with index $\alpha / 2$ and

$$
\theta_{i}^{*}=\Delta_{i} / e^{t^{*}}, \quad i=1,2, \ldots
$$

is such that the sequence of squares $\left(\left(\theta_{1}^{*}\right)^{2},\left(\theta_{2}^{*}\right)^{2}, \ldots\right)$ is distributed according to the Poisson-Dirichlet law $\operatorname{PD}(\alpha / 2,0)$; see Pitman and Yor [14.

We also stress that every coalescent $\mathbf{C}^{\theta}$ can be obtained as a limit of appropriate caravan parking problems, which are quite natural given the results of [3, 5]. Precisely, suppose that a sequence of probabilities $\mathbf{p}^{n}=\left(p_{1}^{n}, \ldots, p_{m_{n}}^{n}\right)$ satisfying $p_{1}^{n} \geq \ldots \geq p_{m_{n}}^{n}>0$ is given, and satisfies

$$
\max _{1 \leq i \leq m_{n}} p_{i}^{n} \underset{n \rightarrow \infty}{\rightarrow} 0 \quad \text { and } \quad \sigma\left(\mathbf{p}^{n}\right)^{-1} p_{i}^{n} \underset{n \rightarrow \infty}{\rightarrow} \theta_{i} \quad i \geq 1
$$

for a sequence $\boldsymbol{\theta}$ as described above, and where $\sigma(\mathbf{p})=\sqrt{\sum_{i=1}^{m} p_{i}^{2}}$ when $\mathbf{p}=\left(p_{1}, \ldots, p_{m}\right)$. For every $n$, let $\tau_{n}$ be a uniform permutation on $\left\{1,2, \ldots, m_{n}\right\}$. Consider the parking problem where the caravans which try to park successively have magnitudes $p_{\tau_{n}(1)}^{n}, p_{\tau_{n}(2)}^{n}, \ldots$ Let $U_{1}, U_{2}, \ldots$ be independent uniform $(0,1)$ random variables independent of $\tau_{n}$, so we may consider the bridge with exchangeable increments

$$
B^{(n)}(s)=\sigma\left(\mathbf{p}^{n}\right)^{-1}\left(-x+\sum_{i=1}^{m_{n}} p_{\tau_{n}(i)}^{n} \mathbb{1}_{\left\{s \geq U_{i}\right\}}\right), \quad 0 \leq s \leq 1 .
$$

Kallenberg's theorem shows that under the asymptotic assumptions on $\mathbf{p}^{n}, B^{(n)}$ converges in distribution to the bridge $B^{\theta}$ defined above.

Now for $t \geq 0$, let $I_{t}^{n}=\inf \left\{i \geq 1: \sum_{j=i+1}^{m_{n}} p_{\tau_{n}(j)}^{n} \leq t\right\}$. The following analogue of Lemma 5 holds.

Lemma 7 For every $t \geq 0$, the process

$$
\sigma\left(\mathbf{p}^{n}\right)^{-1} \sum_{i=I_{t}^{n}+1}^{m_{n}} p_{\tau_{n}(i)}^{n} \mathbb{1}_{\left\{s \geq U_{i}\right\}}, \quad 0 \leq s \leq 1
$$

converges in probability for the uniform norm to the pure drift $s \mapsto t s$ as $n \rightarrow \infty$. 
Proof. The key to this lemma is to show that

$$
\max _{i \geq I_{t}^{n}} \sigma\left(\mathbf{p}^{n}\right)^{-1} p_{\tau_{n}(i)}^{n} \rightarrow 0
$$

in probability as $n \rightarrow \infty$. The result is then obtained via the so-called "weak law of large numbers for sampling without replacement": if $x_{i}^{n}, 1 \leq i \leq n$ is a sequence with sum $t$ satisfying $\max _{1 \leq i \leq n} x_{i}^{n} \rightarrow 0$ as $n \rightarrow \infty$, and if $\tau_{n}$ is a uniform permutation on $\{1, \ldots, n\}$, then for every rational $r \in[0,1], \sum_{i=1}^{n} x_{\tau_{n}(i)}^{n} \mathbb{1}_{\left\{r \geq U_{i}\right\}} \rightarrow t r$ in probability (in fact in $L^{2}$ ). The result in probability remains true if $x_{i}^{n}, 1 \leq i \leq n$ is random with sum $t$, and $\max _{1 \leq i \leq n} x_{i}^{n} \rightarrow 0$ in probability. One concludes that the process $\left(\sum_{i=1}^{n} x_{\tau_{n}(i)}^{n} \mathbb{1}_{\left\{s \geq U_{i}\right\}}, 0 \leq s \leq 1\right)$ converges in probability to $(t s, 0 \leq s \leq 1)$ for the uniform norm by a monotonicity argument. The lemma is then proved by letting $x_{1}=\sigma\left(\mathbf{p}^{n}\right)^{-1} p_{\tau_{n}\left(I_{t}^{n}+1\right)}^{n}, x_{2}=\sigma\left(\mathbf{p}^{n}\right)^{-1} p_{\tau_{n}\left(I_{t}+2\right)}^{n}, \ldots, x_{m_{n}-I_{t}^{n}}=\sigma\left(\mathbf{p}^{n}\right)^{-1} p_{\tau_{n}\left(m_{n}\right)}^{n}, x_{m_{n}-I_{t}^{n}+1}=$ $t-\sum_{i=I_{t}^{n}+1}^{m_{n}} \sigma\left(\mathbf{p}^{n}\right)^{-1} p_{\tau_{n}\left(I_{t}^{n}+1\right)}^{n}$ (note that this last term is $\leq \sigma\left(\mathbf{p}^{n}\right)^{-1} p_{\tau_{n}\left(I_{t}^{n}\right)}^{n}$, which goes to $0)$.

So let us show (13). To this end, let $0<\rho<1$, then $X_{n}^{\rho}:=\sum_{i=\left\lfloor\rho m_{n}\right\rfloor}^{m_{n}} \sigma\left(\mathbf{p}^{n}\right)^{-1} p_{\tau_{n}(i)}^{n} \rightarrow$ $\infty$ in probability, since $E\left[X_{n}^{\rho}\right] \sim \sigma\left(\mathbf{p}^{n}\right)^{-1}(1-\rho)$ goes to infinity (notice $\sigma(\mathbf{p}) \leq p_{1}$ ) while $E\left[\left(X_{n}^{\rho}\right)^{2}\right] \sim E\left[X_{n}^{\rho}\right]^{2}$, as a simple computation shows. Therefore, $I_{t}^{n} \sim m_{n}$ in probability. Consequently, for any $K \in \mathbb{N}$, the quantity $P\left(\tau_{n}^{-1}(1)<I_{t}^{n}, \ldots, \tau_{n}^{-1}(K)<I_{t}^{n}\right)$ goes to 1 , so $\min _{i \geq I_{t}^{n}} \tau_{n}(i) \rightarrow \infty$ in probability. But then, for any $\varepsilon>0$, if $K$ is such that $\theta_{K}<\varepsilon / 2$, then $\sigma\left(\mathbf{p}^{n}\right)^{-1} p_{K}^{n} \leq \varepsilon$ for $n$ large. Up to taking $n$ even larger, with probability close to 1 , $\tau_{n}(i) \geq K$ for $i \geq I_{t}^{n}$ and therefore $\max _{i \geq I_{t}^{n}} \sigma\left(\mathbf{p}^{n}\right)^{-1} p_{\tau_{n}(i)}^{n} \leq \varepsilon$, hence (13).

One deduces, as around the proof of Proposition 1 the following claim. Let $\mathbf{X}^{(n)}(t)=$ $\Lambda^{\mathbf{p}^{n} \circ \tau_{n}}\left(I_{t}^{n}\right)$ be as above with data $m=m_{n}, p_{\tau_{n}(i)}^{n}, 1 \leq i \leq m_{n}, s_{i}=U_{i}$. Then

Proposition 2 As $n \rightarrow \infty$, under the asymptotic regime (19), the process $\left(\mathbf{X}^{(n)}(t), t \geq 0\right)$ converges in the sense of weak convergence of finite-dimensional marginals to the timereversed eternal additive coalescent $\left(\mathbf{C}^{\theta}(-\log (t)), t \geq 0\right)$.

\subsection{On the marginal distributions}

It would also be interesting to determine the marginal laws of the fragmentation $\mathbf{F}^{(\alpha)}(t):=$ $\mathbf{C}^{(\alpha)}(-\log t)$. The task seems quite difficult if started from the description of $\mathbf{F}^{(\alpha)}(t)$ in terms of lengths of constancy intervals of Vervaat transform of bridges, because excursion theory seems powerless here, unlike in [13]. In particular, the fact that the fragmentation is based on stable loops and not stable bridges impedes the application of results of Miermont 13. on additive coalescents based on bridges of certain Lévy processes.

Another way to start the exploration is to use the representation of fragmentation processes $\mathbf{F}^{\theta}(t):=\mathbf{C}^{\theta}(-\log t)$ described in the preceding section with the help of Inhomogeneous Continuum Random Trees (ICRT) discussed in [3]. In particular, it is easy to obtain the first moment of a size-biased $\operatorname{pick}^{2} \mathbf{F}_{\dagger}(t)$ from the sequence $\mathbf{F}(t)$ for any fixed $t$, as follows.

Let us recall the basic facts on the $\operatorname{ICRT}(\boldsymbol{\theta})$ construction of $\mathbf{F}^{\theta}$. The ICRT can be viewed via a stick-breaking construction as the metric completion of the positive real line

\footnotetext{
${ }^{2}$ Recall that a size-biased pick $X_{\dagger}$ from a (random) positive sequence $\left(X_{i}, i \geq 1\right)$ with sum $0<S<\infty$ a.s. is a random variable of the form $X_{i^{*}}$, where $P\left(i^{*}=i \mid X_{j}, j \geq 1\right)=X_{i} / S$.
} 
$\mathbb{R}_{+}$endowed with a non standard metric. Precisely, suppose we are given the following independent random elements:

- A Poisson process $\left\{\left(U_{i}, V_{i}\right), i \geq 1\right\}$ on the octant $\mathbb{O}=\{(x, y): 0<y<x\} \subset \mathbb{R}_{+}^{2}$, with intensity $\theta_{0} \mathrm{~d} x \mathrm{~d} y \mathbb{1}_{\mathbb{O}}$, so in particular $\left\{U_{i}, i \geq 1\right\}$ is a Poisson process with intensity $\theta_{0} x \mathrm{~d} x \mathbb{1}_{x \geq 0}$,

- A sequence of independent Poisson processes $\left\{\xi_{i, j}, j \geq 1\right\}, i=1,2, \ldots$ with respective intensities $\theta_{i} \mathrm{~d} x \mathbb{1}_{x \geq 0}, i=1,2, \ldots$.

We distinguish the points $\left(V_{i}, i \geq 1\right),\left(\xi_{i, 1}, i \geq 1\right)$ as joinpoints, while $\left(U_{i}, i \geq 1\right),\left(\xi_{i, j}, i \geq\right.$ $1, j \geq 2)$ are called cutpoints. If $\eta$ is a cutpoint, let $\eta^{*}$ be its associated joinpoint, i.e. $U_{i}^{*}=V_{i}, \xi_{i, j}^{*}=\xi_{i, 1}$. By the assumption on $\boldsymbol{\theta}$, it is a.s. possible to arrange the cutpoints by increasing order $0<\eta_{1}<\eta_{2}<\ldots$. We then construct a family $\mathcal{R}(k), k \geq 1$ of "reduced trees" as follows. Cut the set $(0, \infty)$ into "branches" $\left(\eta_{i}, \eta_{i+1}\right]$, where by convention $\eta_{0}=0$. Let $\mathcal{R}(1)$ be the segment $\left(0, \eta_{1}\right]$, endowed with the usual distance $d_{1}(x, y)=|x-y|$. Then given $\mathcal{R}(k), d_{k}$, we obtain $\mathcal{R}(k+1)$ by adding the branch $\left(\eta_{k}, \eta_{k+1}\right]$ somewhere on $\mathcal{R}(k)$, and we plant the left-end $\eta_{k}$ on the joinpoint $\eta_{k}^{*}$ (since a.s. $\eta^{*}<\eta$, the point $\eta_{k}^{*}$ is indeed an element of $\mathcal{R}(k))$. Precisely, $\mathcal{R}(k+1)=\left(0, \eta_{k+1}\right]$ and $d_{k+1}(x, y)=d_{k}(x, y)$ if $x, y \in \mathcal{R}(k), d_{k+1}(x, y)=|x-y|$ if $x, y \in\left(\eta_{k}, \eta_{k+1}\right]$, and $d_{k+1}(x, y)=x-\eta_{k}+d_{k}\left(y, \eta_{k}^{*}\right)$ if $x \in\left(\eta_{k}, \eta_{k+1}\right], y \in \mathcal{R}(k)$. As the distances $d_{k}$ are compatible by definition, this defines a random metric space $(0, \infty), d$ such that the restriction of $d$ to $\mathcal{R}(k)$ is $d_{k}$, we call its metric completion $\mathcal{T}^{\theta}$ the $\operatorname{ICRT}(\boldsymbol{\theta})$, its elements are called vertices. The point $\varnothing=\lim _{n \rightarrow \infty} 1 / n$ is distinguished and called the root.

One can see that $\mathcal{T}^{\theta}$ is an $\mathbb{R}$-tree, i.e. a complete metric space such that for any $x, y \in \mathcal{T}^{\theta}$ there is a unique simple path $[[x, y]]$ from $x$ to $y$, which is isometric to the segment $[0, d(x, y)]$, i.e. is a geodesic. Moreover, it can be endowed with a natural measure $\mu^{\theta}$ which is the weak limit as $n \rightarrow \infty$ of the empirical measures $n^{-1} \sum_{i=1}^{n} \delta_{\eta_{i}}$. This measure is non-atomic and supported on leaves, i.e. vertices $x \in \mathcal{T}^{\theta}$ such that $x \notin[[\varnothing, y]] \backslash\{y\}$ for any $y \in \mathcal{T}^{\theta}$. Non-leaf vertices form a set called the skeleton. A second natural measure is the Lebesgue measure $\lambda$ on $\mathcal{T}^{\theta}$, i.e. the unique measure such that $\lambda([[x, y]])=d(x, y)$ for any $x, y$, and this measure is supported on the skeleton.

Now for each $t$ consider a Poisson measure on $\mathcal{T}^{\theta}$ with atoms $\left\{x_{i}^{t}, i \geq 1\right\}$, with intensity $t \lambda(\mathrm{d} x)$, so the different processes are coupled in the natural way as $t$ varies, i.e. $\left\{x_{i}^{t}, i \geq 1\right\}$ increases with $t$. These points disconnect the tree into a forest of disjoint connected tree components, order them as $\mathcal{F}_{i}^{\theta}(t), i \geq 1$ by decreasing order of $\mu^{\theta}$-mass. Then the process $\left(\left(\mu^{\theta}\left(\mathcal{F}_{i}^{\theta}(t)\right), i \geq 1\right), t \geq 0\right)$ of these $\mu^{\theta}$-masses has same law as $\mathbf{F}^{\theta}$. A size-biased pick from this sequence of masses is then obtained as the $\mu^{\theta}$-mass of the tree component at time $t$ that contains an independent $\mu^{\theta}$-sample, conditionally on $\left(\mathcal{T}^{\theta}, \mu^{\theta}\right)$. Therefore, if $\mathbf{F}_{\dagger}^{\theta}(t)$ denotes such a size-biased pick, $E\left[\mathbf{F}_{\dagger}^{\theta}(t)\right]$ is the probability that two independent $\mu^{\theta}$ samples $X_{1}, X_{2}$ belong to the same tree component of the cut tree, i.e. that no atom of the Poisson measure at time $t$ falls in the path $\left[\left[X_{1}, X_{2}\right]\right]$, and hence it equals $E\left[e^{-t d\left(X_{1}, X_{2}\right)}\right]$.

It turns out [3] that $d\left(X_{1}, X_{2}\right)$ has same law as the length $\eta_{1}$ of the first branch (i.e. the length of $\mathcal{R}(1)$ ). It is easy to see (see also [7]) that this branch's length has law

$$
P\left(\eta_{1}>r\right)=e^{-\theta_{0}^{2} r 2 / 2} \prod_{i=1}^{\infty}\left(1+\theta_{i} r\right) e^{-\theta_{i} r} .
$$


In our setting, recall that the random sequence $\theta^{*}$ is related to that of the atoms $\left(\Delta_{i}\right)$ of a Poisson measure on $(0, \infty)$ with intensity $\frac{\alpha(\alpha-1)}{\Gamma(2-\alpha)} x^{-1-\alpha} d x$ by (10) and (11). Observe that $\mathbf{F}^{(\alpha)}(t)=\mathbf{F}^{\theta^{*}}\left(t / e^{t^{*}}\right)$, and since we must take a Poisson process with intensity $t / e^{t^{*}}$ on the skeleton of the $\operatorname{ICRT}\left(\theta^{*}\right)$, terms $e^{t^{*}}$ cancel out and $E\left[\mathbf{F}_{\dagger}^{(\alpha)}(t)\right]=E\left[e^{-t \eta}\right]$ where

$$
\begin{aligned}
P(\eta \geq r) & =E\left[\prod_{i=1}^{\infty}\left(1+r \Delta_{i}\right) e^{-r \Delta_{i}}\right] \\
& =\exp \left(-\int_{0}^{\infty} \frac{\alpha(\alpha-1) \mathrm{d} x}{\Gamma(2-\alpha) x^{1+\alpha}}(1-\exp (-r x+\log (1+r x)))\right) \\
& =\exp \left(-(\alpha-1) r^{\alpha}\right),
\end{aligned}
$$

which is a Weibull distribution. This gives (at least in principle) the first moment

$$
E\left[\mathbf{F}_{\dagger}^{(\alpha)}(t)\right]=\int_{0}^{\infty} \alpha(\alpha-1) r^{\alpha-1} \exp \left(-t r-(\alpha-1) r^{\alpha}\right) \mathrm{d} r .
$$

In principle, this method could be used for the computation of moments of higher order, where the length $\eta_{1}$ of the first branch would simply be replaced by the total length $\eta_{k}$ of $\mathcal{R}(k)$. Unfortunately, the distribution of $\eta_{k}$ is complicated for $k=3$, and seems intractable for higher $k$ 's $([2])$.

\section{References}

[1] David J. Aldous and Jim Pitman. The standard additive coalescent. Ann. Probab., 26(4):1703-1726, 1998.

[2] David J. Aldous and Jim Pitman. A family of random trees with random edge lengths. Random Structures Algorithms, 15(2):176-195, 1999.

[3] David J. Aldous and Jim Pitman. Inhomogeneous continuum random trees and the entrance boundary of the additive coalescent. Probab. Theory Relat. Fields, 118(4):455-482, 2000.

[4] Jean Bertoin. A fragmentation process connected to Brownian motion. Probab. Theory Relat. Fields, 117(2):289-301, 2000.

[5] Jean Bertoin. Eternal additive coalescents and certain bridges with exchangeable increments. Ann. Probab., 29(1):344-360, 2001.

[6] Jean Bertoin. Random covering of an interval and a variation of Kingman's coalescent. Random Structures Algorithms, 25(2):277-292, 2004.

[7] Michael Camarri and Jim Pitman. Limit distributions and random trees derived from the birthday problem with unequal probabilities. Electron. J. Probab., 5:no. 1, 18 pp. (electronic), 2000.

[8] Philippe Chassaing and Guy Louchard. Phase transition for parking blocks, Brownian excursion and coalescence. Random Structures Algorithms, 21(1):76-119, 2002. 
[9] Aryeh Dvoretzky and Herbert E. Robbins. On the "parking" problem. Magyar Tud. Akad. Mat. Kutató Int. Közl., 9:209-225, 1964.

[10] Steven N. Evans and Jim Pitman. Construction of Markovian coalescents. Ann. Inst. Henri Poincare Probab. Stat., 34(3):339-383, 1998.

[11] Olav Kallenberg. Canonical representations and convergence criteria for processes with interchangeable increments. Z. Wahrscheinlichkeitstheorie und Verw. Gebiete, $27: 23-36,1973$.

[12] Jean-François Le Gall. Spatial branching processes, random snakes and partial differential equations. Lectures in Mathematics ETH Zürich. Birkhäuser Verlag, Basel, 1999.

[13] Grégory Miermont. Ordered additive coalescent and fragmentations associated to Levy processes with no positive jumps. Electron. J. Probab., 6:no. 14, 33 pp. (electronic), 2001.

[14] Jim Pitman and Marc Yor. The two-parameter Poisson-Dirichlet distribution derived from a stable subordinator. Ann. Probab., 25(2):855-900, 1997.

[15] Alfréd Rényi. On a one-dimensional problem concerning random space filling. Magyar Tud. Akad. Mat. Kutató Int. Közl., 3(no 1/2):109-127, 1958. 